\title{
Chikungunya infection: de-linking replication from symptomatology reveals the central role of muscle
}

\begin{abstract}
Anne Moscona
Chikungunya virus (CHIKV) is an emerging arbovirus, endemic in many parts of the world, that is spread by travelers and adapts to new mosquito vectors that live in temperate climates. CHIKV replicates in many host tissues and initially causes a self-limiting febrile illness similar to dengue. However, in $30 \%-40 \%$ of cases, CHIKV also causes long-term painful and debilitating muscle and joint pain, the pathogenesis of which remains unknown. In this issue of the $J C l$, Lentscher et al. engineered a skeletal muscle-restricted CHIKV to show that while musculoskeletal disease requires viral replication in affected muscle, muscular pathology is mediated by host immunological factors. These findings de-link viral replication and disease symptoms, illuminate the virus-host interplay in CHIKV symptomatology, and raise the possibility that immune modulation is a therapeutic option. The results also highlight possible solutions to existing vaccine barriers and provide insights that may apply to other viral diseases.
\end{abstract}

Department of Pediatrics, Microbiology \& Immunology, and Department of Physiology \& Cellular Biophysics, Columbia University College of Physicians \& Surgeons, New York, New York, USA
Chikungunya is a uniquely dangerous emerging virus

Chikungunya virus (CHIKV) is a member of the Old World Alphavirus genus of the Togaviridae family. It is an arbovirus that causes widespread disease in the developing world and an increasing number of outbreaks in temperate climates. The virus is small (70 $\mathrm{nm}$ ) and enveloped, carrying a singlestranded, message-sense RNA genome of about 11,800 nucleotides. Several features set CHIKV apart from related mosquito-borne febrile disease-causing viruses, making its emergence a major global concern. Its high rates of infection and alternating rural and urban transmission cycles mean that it spreads quickly and across broad geographic areas. While several emerging viruses are expanding in range as a result of climate and socioenvironmental changes that affect transmission, CHIKV expansion is aided by the capacity of the virus to adapt to additional mosquito vectors that live in tem- perate climates (1-4), the high burden of disease caused by large urban outbreaks, and the fact that infected humans can seed transmission in naive areas, setting up transmission and new outbreaks (5-7). CHIKV is rapidly establishing a stronghold in a widening geographic range, at a time when there are no effective antivirals or available vaccines and a lack of understanding of the basis for the severe long-term sequelae of infection. Moreover, while CHIKV infection initially causes a self-limiting febrile illness similar to that of dengue, in $30 \%-40 \%$ of cases it leads to a persistent, painful, and debilitating rheumatic disease (8). The pathogenesis of these long-term musculoskeletal symptoms after CHIKV infection is not understood.

\section{Three strides toward pathogenesis, and a potential therapy}

In this issue of the JCI, Lentscher et al. clearly define a role for $\mathrm{CHIKV}$ replication

Related Article: p. 1466

Conflict of interest: The author has declared that no conflict of interest exists.

Copyright: @ 2020, American Society for Clinical Investigation.

Reference information: / Clin Invest. 2020;130(3):1099-1101. https://doi.org/10.1172/JCI134746. 
that particular restrictive miRNA-in this case, the skeletal muscle. The researchers incorporated sequences complementary to skeletal muscle-specific miRNA miR206 into the CHIKV genome, generating a skeletal muscle-restricted CHIKV strain (SKE) along with a mismatch sequence control CHIKV strain (SKE MM). These viruses proved to be perfect tools. SKE was specifically restricted by miR206 in culture and restricted in replication in only the skeletal muscle in mice (while replicating normally at other sites); on the other hand, SKE MM showed neither restriction. Armed with these tools, the authors examined the specific impact of infection at this key site. This is an approach that could be applied, as the authors note, to other sites of CHIKV replication, but also to a range of other viral illnesses for which the precise sites of replication associated with disease are enigmatic (9).

\section{Replication in muscle is necessary for muscle disease}

CHIKV spotlights a puzzle for a broad swath of viral diseases; the virus infects a variety of cell types and replicates at many sites within the host, but disease is tissue specific. The general concept of a requisite interplay between host and pathogen in pathogenesis is often mentioned in the literature but only rarely elucidated. The two viruses differing only in being muscle restricted (SKE) or permissive (SKE MM) were similar in their induction of viral load and tissue burden of infection in mice in every way but one: musculoskeletal disease (muscle inflammation) occurred only with SKE MM. Replication in muscle is required for disease, but it is not enough to elicit pathology (9).

\section{Necessary but not sufficient: host inflammation mediates muscle disease}

The mice infected with SKE virus (restricted in skeletal muscle cells) had less inflammation, $\mathrm{T}$ cell infiltration into the interosseous muscle (the site of muscle disease in this model), and inflammatory mediators at the site, but only in the local skeletal muscle. SKE MM infection recruited the inflammatory mediators in the muscle seen in humans with CHIKV, including IL-6 (9). However, the global inflammatory picture in the mice outside the interosseous muscle was no different after infection with the two viruses. Pathogenesis was truly local. The authors were then perfectly positioned to ask: is disease due to viral replication or due to the influx of inflammatory mediators? Remarkably, blocking IL- 6 with receptor blocking antibody ablated disease in the muscle. Even in the face of rampant SKE MM viral replication in the muscle, without IL-6 there was limited tissue disease. As the authors note, the question solved here for CHIKV also applies to other viruses that induce myositis, including influenza, where the relative contributions of viral replication and the host immunologic response to the tissue damage are unknown. The tissue restriction strategies used here may be applicable to other tissue-specific diseases as well (9). Moreover, the finding that IL-6 is key to disease induction and the possibility that an existing anti-IL-6 antibody (12) might ameliorate disease in humans is an exciting example of therapy based on disease pathogenesis, lightening the standard focus on targeting solely viral factors for therapies.

\section{CHIKV vaccines: a valuable attenuation strategy?}

While a CHIKV vaccine should be a public health priority, its development has to overcome the difficulties associated with multiple strains, unpredictable outbreaks, and vaccine-induced symptoms $(13,14)$. The findings in Lentscher et al. may provide a solution to precisely this latter problem (9). A significant question that emerges from this work is whether a vaccine from engineered SKE virus, or a derivative of it, would protect. Although there are no licensed therapies or vaccines to date, over the last ten years progress has been made toward several vaccine candidates (15). Live vaccines would be ideal in terms of effective and long-lasting crossCHIKV clade protection, but they would face the challenge of achieving the right balance between viral attenuation (dampening of viral replication) and immunity induction (eliciting a protective response). Attenuation must achieve safety yet generate enough antigen to elicit protective immunity. The first promising vaccine candidate, a live attenuated strain generated by tissue culture passage (16), was highly immunogenic in phase II trials, but was derailed because it caused transient musculoskeletal symptoms in $8 \%$ of vaccinees (17) and the attenuation was unstable, with frequent reversion at the attenuating sites $(18,19)$. Newer attenuated CHIKV vaccine candidates include one bearing an internal ribosome entry site (IRES) replacing the CHIKV subgenomic promoter, preventing replication in the mosquito vector and attenuating replication in humans while protecting nonhuman primates against challenge $(20,21)$. Other candidate strategies under hopeful consideration include a nonreplicating virus-like particle assembled from engineered CHIKV proteins $(22,23)$, a recombinant live-attenuated measles virus vectored vaccine expressing CHIKV structural proteins (24), and a recombinant Eilat/CHIKV chimeric virus that is replication defective in mosquitoes and protective in nonhuman primates (25). The property conferred by the miRNA complementary sequences in the SKE CHIKV strain described by Lentscher et al.-restriction of replication in skeletal muscle-could eliminate the concern that live virus vaccines may target skeletal muscle and produce disease (9). These viruses will replicate enough to induce immunity without risking the hallmark disease manifestations. Replication, de-linked from disease symptoms, could then be modulated by additional attenuating mutations, as desired, to achieve an optimal level of immune response. Beyond CHIKV, it is tempting to consider whether engaging the miRNA system to restrict viral replication in specific target tissues may be a broader attenuation strategy for viral vaccine candidates.

Address correspondence to: Anne Moscona, 701 West 168th Street, New York, New York, 10032, USA. Phone: 347.366.2459; Email: am939@cumc.columbia.edu.

1. Nunes MR, et al. Emergence and potential for spread of Chikungunya virus in Brazil. BMC Med. 2015;13:102.

2. Kraemer MU, et al. The global distribution of the arbovirus vectors Aedes aegypti and Ae. albopictus. Elife. 2015;4:e08347.

3. Tsetsarkin KA, Chen R, Weaver SC. Interspecies transmission and chikungunya virus emergence. Curr Opin Virol. 2016;16:143-150.

4. Tsetsarkin KA, et al. Multi-peaked adaptive landscape for chikungunya virus evolution predicts continued fitness optimization in Aedes albopictus mosquitoes. Nat Commun. 2014;5:4084. 
5. Gibney KB, et al. Chikungunya fever in the United States: a fifteen year review of cases. Clin Infect Dis. 2011;52(5):e121-e126.

6. Rezza G, et al. Infection with chikungunya virus in Italy: an outbreak in a temperate region. Lancet. 2007;370(9602):1840-1846.

7. Grandadam M, et al. Chikungunya virus, southeastern France. Emerging Infect Dis. 2011;17(5):910-913.

8. Suhrbier A, Jaffar-Bandjee MC, Gasque P. Arthritogenic alphaviruses--an overview. Nat Rev Rheumatol. 2012;8(7):420-429.

9. Lentscher AJ, et al. Chikungunya virus replication in skeletal muscle cells is required for disease development. J Clin Invest. 2020;130(3):1466-1478.

10. Martinez J, Patkaniowska A, Urlaub H, Lührmann R, Tuschl T. Single-stranded antisense siRNAs guide target RNA cleavage in RNAi. Cell. 2002;110(5):563-574.

11. Landgraf $\mathrm{P}$, et al. A mammalian microRNA expression atlas based on small RNA library sequencing. Cell. 2007;129(7):1401-1414.

12. Scott LJ. Tocilizumab: A review in rheumatoid arthritis. Drugs. 2017;77(17):1865-1.8.7.9.

13. Silva LA, Dermody TS. Chikungunya virus: epidemiology, replication, disease mechanisms, and prospective intervention strategies. J Clin Invest. 2017;127(3):737-749.

14. Langsjoen RM, et al. Chikungunya virus strains show lineage-specific variations in virulence and cross-protective ability in murine and nonhuman primate models. mBio. 2018;9(2):e02449-17.

15. Rezza G, Weaver SC. Chikungunya as a paradigm for emerging viral diseases: Evaluating disease impact and hurdles to vaccine development. PLoS Negl Trop Dis. 2019;13(1):e0006919.

16. Levitt NH, Ramsburg HH, Hasty SE, Repik PM, Cole FE, Lupton HW. Development of an attenuated strain of chikungunya virus for use in vaccine production. Vaccine. 1986;4(3):157-162.

17. Edelman R, Tacket CO, Wasserman SS, Bodison SA, Perry JG, Mangiafico JA. Phase II safety and immunogenicity study of live chikungunya virus vaccine TSI-GSD-218. Am J Trop Med Hyg. 2000;62(6):681-685.

18. Hoke $\mathrm{CH}$, et al. US military contributions to the global response to pandemic chikungunya. Vaccine. 2012;30(47):6713-6720.

19. Gorchakov R, et al. Attenuation of chikungunya virus vaccine strain $181 /$ clone 25 is determined by two amino acid substitutions in the E2 envelope glycoprotein. J Virol. 2012;86(11):6084-6096.
20. Plante KS, Rossi SL, Bergren NA, Seymour RL, Weaver SC. Extended preclinical safety, efficacy and stability testing of a live-attenuated chikungunya vaccine candidate. PLoS Negl Trop Dis. 2015;9(9):e0004007.

21. Roy CJ, et al. Chikungunya vaccine candidate is highly attenuated and protects nonhuman primates against telemetrically monitored disease following a single dose. J Infect Dis. 2014;209(12):1891-1899.

22. Akahata $W$, et al. A virus-like particle vaccine for epidemic Chikungunya virus protects nonhuman primates against infection. Nat Med. 2010;16(3):334-338.

23. Chang LJ, et al. Safety and tolerability of chikungunya virus-like particle vaccine in healthy adults: a phase 1 dose-escalation trial. Lancet. 2014;384(9959):2046-2052.

24. Ramsauer K, et al. Immunogenicity, safety, and tolerability of a recombinant measles-virusbased chikungunya vaccine: a randomised, double-blind, placebo-controlled, active-comparator, first-in-man trial. Lancet Infect Dis. 2015;15(5):519-527.

25. Erasmus JH, et al. A chikungunya fever vaccine utilizing an insect-specific virus platform. Nat Med. 2017;23(2):192-199. 